\title{
¿Control o autonomía? El debate sobre agencias regulatorias independientes
}

\author{
Diego Pardow Lorenzo*
}

\begin{abstract}
RESUMEN
Chile se encuentra en su tercer proceso de reformas institucionales en menos de un siglo. Al igual que las anteriores, las reformas actuales comparten una vocación internacionalista y tecnocrática. Estos dos procesos previos, sin embargo, tienen también una importante diferencia. Mientras las reformas impulsadas por la misión Kemmerer durante los años 20 fueron graduales, la "revolución silenciosa" de la década de los 80 fue el complemento de un programa acelerado de privatizaciones. Las agencias creadas durante las reformas kemmererianas fueron moviéndose progresivamente hacia un diseño con altos estándares de independencia formal y un sistema funcional de distribución de competencia. Al contrario, las reformas de los Chicago boys establecieron una autoridad regulatoria diferenciada para cada uno de los mercados que pasaron a liberalizarse. Ello generó un sistema atomizado y con bajos niveles de independencia formal. En este contexto, es importante que el aumento en los niveles de independencia formal de las agencias venga acompañado de una racionalización de sus competencias regulatorias y un fortalecimiento de los mecanismos de coordinación administrativa.
\end{abstract}

Agencias regulatorias - independencia formal - diseño institucional

Autonomy or control?

The debate regarding Independent Regulatory Agencies

\begin{abstract}
Chile is undergoing its third process of institutional reforms in about a century. Similarly than the previous reforms, current ones share an internationalist and technocratic approach. These previous two processes of reforms, however, also have an important difference. Whereas the
\end{abstract}

* Licenciado en Ciencias Jurídicas y Sociales, Universidad de Chile. Doctor en Derecho, Universidad de California, Estados Unidos. Profesor de Derecho, Facultad de Derecho, Universidad de Chile. Correo electrónico: dpardow@derecho.uchile.cl

La investigación detrás de este trabajo fue realizada en el marco de un proyecto del Fondo Nacional de Investigación Científica y Tecnológica de Chile (No. 1150181). Agradezco enormemente la colaboración de mis ayudantes Danae Fenner y Alejandra Corvalán.

Artículo recibido el 5 de julio de 2017 y aceptado para su publicación en este número el 23 de julio de 2018 
reforms proposed by the Kemmerer mission during the 1920's were gradually implemented, the "silent revolution" of the 1980's was a complement for an accelerated program of privatizations. The agencies created during the kemmererian reforms were progressively moving towards higher standards of formal independence and within a functional system for distributing jurisdiction. On the contrary, the Chicago boys' reforms established a different regulatory agency for each privatized market. This created an atomized system with lower levels of formal independence. From this perspective, it is important that future increases in the levels of formal independence are coupled with a rationalization of the agencies' regulatory jurisdiction and with stronger mechanisms of administrative coordination.

Regulatory agencies - formal independence - istitutional design

La posibilidad de la autonomía administrativa parece, a primera vista, incompatible con la idea de la unidad del Estado

Jellinek (2012) 633

\section{UNA HISTORIA QUE SE REPITE}

E n el ámbito de la regulación económica, cada cierto tiempo se reitera la importancia de fortalecer la autonomía política de nuestras agencias, así como la calidad técnica de sus decisiones ${ }^{1}$. Por un lado, las autoridades que dependen del ciclo electoral tendrían demasiados incentivos para preocuparse únicamente del corto plazo ${ }^{2}$. Por otro, el carácter técnico de la regulación económica exigiría una burocracia especializada ${ }^{3}$. La independencia surge así como una manera de aislar a las instituciones regulatorias, como una forma de protegerlas frente a las presiones de la política contingente y otorgarles las herramientas necesarias para tomar decisiones de manera objetiva ${ }^{4}$.

${ }^{1}$ En el ámbito de la regulación financiera, por ejemplo, la denominada Comisión Jadresic concluyó que la especificidad técnica de la materia objeto de supervisión exigía mayor autonomía para el regulador, recomendando para estos fines que el superintendente respectivo fuera designado por un periodo fijo. Cerca de 10 años después, la Comisión Desormeaux razonó sobre consideraciones similares, pero esta vez se propuso el reemplazo de la figura unipersonal del superintendente por un consejo colegiado. Finalmente, a comienzos del 2016 la Comisión Cordero reiteró la necesidad de fortalecer la autonomía de los reguladores financieros, enfocando sus propuestas en someter los nombramientos a confirmación por parte del Senado.

2 Wilson, J., Bureaucracy: What government agencies do and why they do it, Basic Book, Nueva York, 1989, pp. 72-82.

${ }^{3}$ La visión tradicional de una organización burocrática se basa precisamente en una idea de especialidad, esto es, limitar las competencias del funcionario para fomentar la especialización en un conjunto limitado de tareas, y así ejercer el poder público de una manera más eficaz. Tratándose de la regulación económica, sin embargo, la demanda por mayor especialización suele venir asociada a una supuesta complejidad de la normativa que afectaría a los sectores relacionados con la energía, las telecomunicaciones o los servicios financieros (v.g. Levy, B. Regulations, institutions and commitment: Comparative studies of telecomunications, Cambridge University Press, Cambridge, 2008.).

${ }^{4}$ Ogus, A., Regulation, legal reform and economic theory, Clarendon Press, Oxford, 1994, pp. 181 y ss. 
El problema es que un conjunto de razones relativamente similares pareciera sostener un conjunto increíblemente diverso de soluciones. En un extremo tenemos casos como el Banco Central, cuya dirección corresponde a un grupo de consejeros, nombrados por un plazo fijo y mediante la intervención de distintos poderes del Estado. En el otro extremo, existen agencias regulatorias como la Subsecretaría de Telecomunicaciones (SUBTEL), cuya dirección recae en una sola persona, nombrada y removida discrecionalmente por el poder ejecutivo. Estas diferencias se repiten en otros ámbitos. En ocasiones incorporamos garantías a nivel constitucional, mientras que en muchas otras nos contentamos con descentralizar legalmente un servicio público ${ }^{5}$. A veces el control presupuestario está concentrado en el Ministerio de Hacienda, pero otras veces es la propia agencia quien tiene cierto control sobre sus finanzas. Esta diversidad de soluciones se repite cuando miramos nuestra experiencia reciente. Durante la última reforma a la legislación eléctrica, por ejemplo, se mantuvieron las facultades del Tribunal de la Libre Competencia (TDLC) para designar a los miembros del panel de expertos, consignando que ello era una garantía suficiente de autonomía. Curiosamente, sin embargo, de manera paralela se tramitaba una reforma al DL 211 cuyo mensaje criticaba las reglas respecto de inhabilidades de los miembros del TDLC, precisamente porque no ofrecían garantías suficientes para la independencia del organismo ${ }^{6}$.

Construir mayor coherencia normativa resulta fundamental para enfrentar el proceso de reformas que se asoma en el horizonte ${ }^{7}$. Efectivamente, el ingreso de Chile a la Organización para la Cooperación y Desarrollo Económico (OCDE) abrió una nueva

${ }^{5}$ El rango normativo de las normas atributivas de competencia pareciera ser el elemento decisivo en la clasificación tradicional de nuestra doctrina administrativa (v.g. CARMONA, C., Una aproximación general sobre las superintendencias desde la perspectiva del Derecho, [inédito, en consulta con el autor], Santiago, 2005). De este modo, organismos como el Banco Central o la Contraloría General de la República, cuya existencia está reconocida en la Constitución Política, formarían la categoría de autonomías constitucionales. En contraste, las superintendencias son organismos que únicamente cuentan con una ley orgánica y serían por ello legalmente descentralizados. Por último, existirían casos como los de la Subsecretaría de Telecomunicaciones, que actúan directamente bajo la personalidad jurídica del Fisco y pertenecen a la categoría de servicios centralizados.

${ }^{6}$ Ver, Boletín 9950-03. Lamentablemente, esta incoherencia legislativa es mucho más frecuente de lo que uno quisiera. Por nombrar otro ejemplo, durante el 2008 la Cámara de Diputados decidió crear una nueva superintendencia porque este tipo de diseño institucional permitía "fortalecer la independencia del regulador en el ámbito educativo” (GARCía, J. F., “¿Inflación de superintendencias? Un diagnóstico crítico desde el derecho regulatorio”, en Actualidad Jurídica, Universidad del Desarrollo, vol. XIX, 2009, pp. 327 330). Algunos meses después, sin embargo, esta misma instancia legislativa consignaba que la crisis del sistema de superintendencias exigía reemplazar la Superintendencia de Valores y Seguros (SVS) por un modelo de comisión.

${ }^{7}$ A modo de ejemplo, actualmente están siendo debatidas en el Congreso Nacional reformas orgánicas significativas al Servicio Nacional del Consumidor (SERNAC) y la Superintendencia de Educación. A ello se agregan proyectos de reforma en actual estudio, como sucede con la Superintendencia de Bancos e Instituciones Financieras (SBIF) y SUBTEL; así como reformas aprobadas recientemente y en camino de implementación, como el nuevo Coordinador Nacional en materia eléctrica y la nueva Comisión de Mercados Financieros. Una revisión de las reformas institucionales impulsadas en el último tiempo, puede encontrarse en: http://www. agendaproductividad.cl/wp-content/uploads/sites/22/2014/10/Estudio-Regulatorio-OCDE.pdf 
etapa de reformas institucionales profundas, siendo el tercer proceso de este tipo en menos de un siglo ${ }^{8}$. Los dos procesos anteriores corresponden a las reformas iniciadas por la misión Kemmerer durante los años 20, así como la denominada "revolución silenciosa" que impulsaron los Chicago boys durante la década de los 80 . Con independencia de las diferencias que se exploran más adelante en este trabajo, los tres procesos comparten una misma visión internacionalista y tecnocrática ${ }^{9}$. El internacionalismo se refiere al propósito de las reformas, en estos tres casos su objetivo era adecuar nuestras instituciones a ciertos estándares internacionales, en particular respecto de la manera en que el Estado debía intervenir en la economía. En contraste, la vocación tecnocrática se refleja en la forma de argumentar: la justificación de estos estándares se realiza sobre la base del crecimiento económico o la eficiencia, antes que la legitimidad democrática, la justicia social u otras razones tradicionales en la discusión de políticas públicas ${ }^{10}$.

Este artículo es el primerio de una serie de trabajos dirigidos a racionalizar nuestra experiencia institucional. En concreto, mientras los otros trabajos de la serie formalizan el modelo de análisis y revisan la evidencia concerniente a los mecanismos para el nombramiento de directivos, este artículo busca simplemente enriquecer el marco teórico con que usualmente discutimos acerca del diseño institucional de nuestras agencias regulatorias $^{11}$. Una revisión de nuestra práctica administrativa muestra un entendimiento bastante vago acerca de las ideas de independencia y autonomía. Por un lado, en nuestra comunidad jurídica pareciera que la independencia es siempre deseable, sin que existan mayores desventajas al implementarla. Por otro lado, y según vimos más arriba, sería completamente independiente del contexto y permitiría justificar diseños institucionales con importantes diferencias entre sí. Desde esta perspectiva, la principal contribución del trabajo es destacar que la independencia ciertamente tiene costos, de modo que la decisión

${ }^{8}$ Morandé, F. y Díaz, J. D., "Chile en el club de los países desarrollados: beneficios, oportunidades y desafíos del ingreso a la OCDE”, en Morandé, F. y Díaz, J. D. (eds.), Chile en el club de los países desarrollados, Ediciones Universidad Católica, Santiago, 2010.

${ }^{9}$ V.g. Silva, P., In the name of reason: Technocrats and politics in Chile, PSU Press, Pennsylvania, 2010. Buena parte de la literatura considera que existiría un cuarto proceso de reformas que compartiría esta visión internacionalista y tecnocrática: la denominada misión Klein-Saks que tuvo lugar durante la década de los 50. En términos de la implementación efectiva de sus recomendaciones, sin embargo, la misión KleinSaks fue poco exitosa (v.g. Luders, R., "La misión Klein-Saks, los Chicago boys y la política económica", en Documentos de trabajo, No 411, Instituto de Economía, Pontificia Universidad Católica de Chile, 2012, pp. 1-34.). Por otra parte, las reformas institucionales ocurridas durante esa época se concentran en aquellos aspectos donde existe consenso entre las misiones Kemmerer y Klein-Saks. Finalmente, la mayor parte de estas reformas ocurrieron bajo el liderazgo del presidente Ibáñez, quien también impulsó las reformas institucionales de la década de los 20 durante su primer gobierno. Por todo lo anterior, este trabajo entiende el trabajo de la misión Klein-Saks como parte del proceso de reformas kemmererianas.

${ }^{10}$ V.g. Hunneus, C., "Technocrats and politicians in an authoritarian regime: The 'ODEPLAN boys' and the 'gremialists' in Pinochet's Chile”, en Journal of Latin American Studies, vol. 32, No 2, 2000, pp. 450-461.

${ }^{11}$ Ver. PArdow, D., Understanding Independent Agencies: Political Insulation, Technical Expertise and the Technocrat's Paradox, [en arbitraje, en consulta con el autor], 2016; y, PARdow, D., ¿Técnicos o políticos?: Radiografía del sistema de nombramiento de directivos en agencias regulatorias independientes, en arbitraje, [en arbitraje, en consulta con el autor], 2017. 
acerca de cuánta autonomía entregar a una agencia exige ponderar valores contrapuestos, como sucede con la estabilidad, reglas del juego y la legitimidad democrática, o con la generación de conocimiento específico y la armonía de la regulación en su conjunto.

Cuando se miran desde este marco teórico las reformas institucionales de las décadas de los 80 y 90, es fácil comprender que actuaron como un complemento del programa de privatizaciones y fueron estableciendo una autoridad regulatoria diferenciada para cada uno de los mercados que pasaron a liberalizarse. Este tipo de diseño institucional generó un sistema de silos extremadamente atomizado. Teniendo en cuenta que este es nuestro punto de partida, aumentar los niveles de independencia supondría implementar reformas de inspiración kemmereriana, pero dentro del esquema de distribución de competencias de los Chicago boys. Ello puede traer complicaciones relevantes, como aumentar los espacios para el arbitraje regulatorio, fomentar dinámicas de acción colectiva y descuidar de los aspectos conductuales de la regulación. En virtud de lo anterior, es importante que una mejora en los niveles de independencia formal venga acompañada de una evaluación crítica respecto del sistema de regulación por silos, así como de un fortalecimiento de los mecanismos de coordinación administrativa.

\section{LAS RAZONES DETRÁS DE LA INDEPENDENCIA}

A la hora de aproximarnos al debate acerca del diseño institucional de los organismos regulatorios, es importante distinguir entre las razones que fundamentan la intervención del Estado en la economía, y aquellas que exigen llevar a cabo dicha intervención mediante organismos relativamente independientes. Mientras el primer grupo de razones responde a un debate referente al rol del Estado frente a las fallas del mercado, el segundo grupo sigue la lógica tradicional de la teoría de la burocracia. Utilizando la clásica distinción de Ripert, la primera se preocupa de la empresa privada y los riesgos del capitalismo, mientras la segunda se preocupa de la burocracia pública y los riesgos del populismo ${ }^{12}$. En definitiva, la demanda de intervención regulatoria se apoya en el potencial abuso de la empresa regulada, en circunstancias que es el potencial abuso del regulador lo que apoya la demanda por independencia institucional. Mientras la intervención regulatoria buscaría un balance entre la autonomía privada y el bien común, la creación de agencias independientes pareciera enfocarse simplemente en la eficacia de la función administrativa.

${ }^{12}$ Ripert, G., Aspectos jurídicos del capitalismo moderno, Comares, Granada, 2001, pp. 281. Un ejemplo típico se encuentra en la regulación de los servicios sujetos a fijación de precios (v.g. electricidad, agua potable o telefonía). La intervención regulatoria tiende a justificarse en la existencia de un monopolio natural, esto es, un esquema donde las potestades públicas actúan como un contrapeso del poder de mercado que se entrega al privado. La autonomía del organismo, en contraste, se fundamenta en el conflicto que supone tener una autoridad sujeta a controles democráticos, pero que simultáneamente tenga potestades para fijar tarifas que afectan la vida cotidiana de los electores; y con ello, su probabilidad de resultar electo (STIGLER, G., "The theory of economic regulation", en Bell Journal of Economics and Management Science, vol. II, 1971, pp. 3-21, 1971). 
La literatura pertinente a agencias regulatorias independientes suele analizar los desafíos de un diseño institucional óptimo a partir de la idea de delegación ${ }^{13}$. En este marco conceptual, la agencia regulatoria ocupa la posición de un agente al que se le delega poder para regular una materia en particular ${ }^{14}$. A su vez, el poder ejecutivo o el Congreso ocuparían el lugar de un principal, esto es, de aquel que soporta los riesgos políticos de las decisiones adoptadas por la agencia regulatoria. De acuerdo con el "principio del aliado", el elemento determinante en la extensión de la delegación regulatoria es la cercanía ideológica entre el agente y el principal. En el contexto de un sistema presidencial, por ejemplo, la voluntad del Presidente para delegar un asunto sería proporcional con el nivel de afinidad política que tenga con la agencia que recibe la delegación ${ }^{15}$. Teniendo en cuenta que el Presidente es una autoridad electa, el denominado "principio del aliado" supone que exista discreción para remover a los directivos de la agencia regulatoria, alterar su presupuesto y, en general, establecer otras formas de premios y castigos que aseguren la primacía de las visiones políticas de la mayoría ${ }^{16}$. Desde esta perspectiva, la independencia es una técnica de Derecho Administrativo que limita la capacidad del poder ejecutivo para implementar las políticas de su preferencia. Por tanto, aumentar los niveles de independencia requeriría una justificación especial en atención a sus problemas de legitimidad democrática ${ }^{17}$.

Existen dos tipos de justificaciones diferentes. La primera, y seguramente la más difundida, es la denominada tesis de la estabilidad. Conforme a ella, cuando existe polarización respecto de un tema particular, todas las posiciones políticas en disputa podrían beneficiarse aumentando la independencia de la agencia encargada de regular

${ }^{13}$ V.g. Gersen, J., “Designing agencies”, en Farber, D. and O'Connell, A. J., Cheltenham Research handbook on public choice and public law, Edward Elgar Publishing, Reino Unido, 2010, pp. 333-362.

${ }^{14}$ Como se aprecia, los esquemas de delegación regulatoria siguen la clásica formulación del problema de agencia. Más que un problema en particular, en todo caso, el problema de agencia hace referencia a los costos que supone para el principal fiscalizar y alinear los intereses del agente. En el caso de una relación de delegación regulatoria, los costos de transacción políticos reflejan el tiempo y esfuerzo que deben desplegar el poder ejecutivo o el Congreso para controlar el comportamiento de la agencia (NorTH, D., Institutions, institutional change and economic perfomrance, Cambridge University Press, Cambridge, 1990).

15 Bawn, K., "Political control versus expertise: Congressional choices about administrative procedures", en American Political Science Review, vol. LXXXIX, 1995, pp. 62-73.

${ }^{16}$ En teoría, un sistema electoral bien diseñado permite que las elecciones sean ganadas por quienes representan de mejor manera al votante mediano, esto es, la posición política que se encuentra más cerca de la mayoría de los electores. En este sentido, el renovado vigor de posiciones centralistas en el Derecho Administrativo comparado suele apoyarse en razones de justicia política; o, puesto de otro modo, en razones acerca de la legitimidad que tendrían las autoridades electas para llevar adelante sus plataformas de campaña (Majone, G., "Nonmajoritarian institutions and the limits of democratic governance: a political transaction-cost approach”, en Journal of Institutional and Theoretical Economics, vol. CLVII, 2001, pp. 57-78).

${ }^{17}$ En este sentido, resulta lógico que la independencia regulatoria forme parte de procesos de reforma que comparten una visión internacionalista y tecnocrática. En términos de política pública, ambas visiones tienen una orientación más bien restrictiva acerca de la capacidad de los gobiernos incumbentes para alterar el modelo de desarrollo económico. Ver: SAavedra, E., "El modelo económico-político de Chile: Desarrollo institucional en la encrucijada”, en Economía y Política, Universidad Adolfo Ibáñez, vol. I, 2014, pp. 117-178. 
ese tema, estableciendo así un mecanismo de restricción mutua ${ }^{18}$. Esta es la justificación tradicional de los bancos centrales, donde el ejemplo recurrente muestra dos coaliciones políticas divergentes compitiendo por controlar el poder ejecutivo. La coalición ganadora lógicamente empujará su agenda política lo más lejos posible. Ahora bien, si la siguiente elección genera un cambio, la nueva coalición gobernante empujará un movimiento en sentido opuesto. En la medida que estos cambios políticos radicales generen costos, un acuerdo donde ambas coaliciones se comprometan con una posición intermedia aumentaría el bienestar social. En otras palabras, la autonomía surge como una manera de proteger este compromiso intertemporal, como una manera de moderar la magnitud del cambio político.

El principal problema de la tesis de la estabilidad tiene que ver con su ceguera respecto de los aspectos sustantivos de este compromiso intertemporal. Sus defensores generalmente asumen que la estabilidad se produce sobre la base de un conjunto de ideas que reflejan el punto medio entre las distintas posiciones en disputa. Ahora bien, la efectividad de este supuesto es algo que dista mucho de ser trivial. Un sistema político capturado por alguna de las posiciones en disputa podría permitir que el compromiso de largo plazo sea representativo de uno de los extremos y no de la posición moderada. Desde este escenario, el remedio sería peor que la enfermedad. La ausencia de compromisos básicos acerca de política monetaria, gasto fiscal o carga tributaria, ciertamente erosionan la capacidad de las instituciones para fomentar el desarrollo económico. Pero teniendo en cuenta que el castigo electoral es uno de los mecanismos básicos para controlar los errores de quienes nos gobiernan, descansar en la volatilidad del ciclo político sería preferible a crear una autonomía institucional que se incline siempre hacia el mismo extremo ${ }^{19}$. En definitiva, salvo que exista cierto equilibrio político en la formación de los acuerdos que dan origen a la delegación regulatoria, la autonomía institucional puede terminar acentuando los sesgos del sistema electoral ${ }^{20}$.

Un ejemplo recurrente puede encontrarse en la hoy desaparecida Interstate Commission of Commerce, creada en 1887 y considerada como la primera agencia independiente en

${ }^{18}$ A diferencia del escenario inicial donde el candidato ganador es aquel que se encuentra más cerca del votante mediano, un escenario de polarización suele representarse con una distribución donde existen dos zonas situadas en extremos opuestos del espectro político que congregan a la mayoría de los votantes (v.g. Issacharoff, S. y Miller, L., "Democracy and electoral processes”, en Farber, D. y O'Connel A.J. (eds.), Research handbook on public choice and public law, Edward Elgar, Cheltenham, 2010, pp. 221-258). En este contexto, la elección de una plataforma política moderada deja de ser garantía de éxito y la alternancia en el poder supone cambios políticos significativos.

19 Tirole, J., “The internal organization of government”, en Oxford Economic Papers, 1994, pp. 1-29.

${ }^{20}$ Cuando existe cierta rotación en el poder, el promedio resultante de alternar posiciones extremas todavía permite acercarse a la posición del votante mediano, por lo que el problema de la polarización radicaría en que una varianza relativamente alta sería representativa de los costos asociados al cambio político. La autonomía construida sobre la base de un acuerdo equilibrado permite acercarse a la posición del votante mediano y simultáneamente reducir los costos del cambio político. En contraste, una autonomía sesgada efectivamente reduce la varianza del ciclo electoral en un escenario de polarización, pero solo a expensas de sacrificar la cercanía promedio con la posición mediana. Ver: Mc Cubbins, M., "The legislative design of regulatory structure”, en American Journal of Political Science, vol. XXXIX, No 4, 1985, pp. 721-748. 
EE.UU. La legislación que establecía esta agencia, delegándole facultades para regular y fijar tarifas respecto de los servicios de ferrocarriles, fue aprobada por una mayoría del saliente Partido Demócrata, luego de haber perdido las elecciones y pocos meses antes que la entrante mayoría del Partido Republicano asumiera sus funciones. Lejos de representar un consenso respecto de la posición de ambas coaliciones -los demócratas apoyaban la fijación de precios y los republicanos la libertad tarifaria-, la nueva legislación buscaba limitar la capacidad del gobierno entrante para cambiar la regulación recientemente aprobada por el gobierno saliente. Esto es lo que la literatura denomina una dinámica de atrincheramiento ${ }^{21}$. En definitiva, la decisión acerca de cuánta autonomía entregar a una agencia regulatoria exige ponderar entre dos valores opuestos: estabilidad y legitimidad. Tratándose de consensos reales, el argumento a favor de la estabilidad se sostiene en que la pérdida de legitimidad sería pequeña, toda vez que la política implementada es similar al promedio que resultaría de la alternancia en el poder entre las distintas coaliciones en disputa ${ }^{22}$. Por el contrario, la pérdida de legitimidad es importante cuando la independencia es resultado del atrincheramiento. No solamente existe un desacople inicial entre la voluntad mayoritaria y las políticas implementadas por la agencia, sino que la autonomía limita la capacidad del ciclo electoral para corregir ese desacople.

La segunda justificación corresponde a la tesis de la precisión, según esta, la autonomía es una herramienta para mejorar la calidad técnica de las decisiones regulatorias. A diferencia del formalismo implícito en la tesis de la estabilidad, la precisión efectivamente supondría una mejora sustantiva en el contenido de las políticas implementadas. Ahora bien, es importante distinguir entre autonomía y especialización. Desde una perspectiva tradicional, cualquier organización burocrática aumenta su efectividad al especializarse en un conjunto limitado de tareas y repetirlas sucesivamente ${ }^{23}$. Esta especialización, sin embargo, puede lograrse perfectamente dentro del aparato centralizado del gobierno.

${ }^{21}$ En EE.UU. la evidencia muestra que la tesis del atrincheramiento tiene un poder explicativo importante. Existe una correlación positiva entre la creación de agencias independientes y la posibilidad de una derrota electoral para el gobierno incumbente, así como con la intensidad de las disputas ejecutivo-legislativo y el número de facciones al interior de la coalición gobernante (Wood, D. y BоотнE, J., "Political transaction costs and the politics of administrative design”, en Journal of Politics, vol. LXIV, 2004, pp. 176-202). Por su parte, en Europa la evidencia también muestra que la creación de agencias independientes suele venir acompañada de un aumento en la intensidad de la afiliación partidaria de los directivos de la agencia (GILARDI, F., "Policy credibility and delegation to independent regulatory agencies: A comparative empirical analysis", en Journal of European Public Policy, vol. IX, N 6, 2002, pp. 873-893).

${ }^{22}$ LEwIS, D., Presidents and the politics of agency design: Political insulation in the United States government bureaucracy, 1946-1997, Stanford University Press, Standford, 2004.

${ }^{23}$ En términos de Max Weber, por ejemplo, la especialización de la burocracia es proporcional a su capacidad para ejercer poder (Weber, M., Economía y Sociedad, Fondo de Cultura Económica, México, 1956, pp. 382-394). Con todo, la generación de conocimiento especializado es algo que determina la relación entre el funcionario y el ciudadano, sin que de ello se desprenda una necesidad de fortalecer la autonomía en la relación entre el funcionario y el gobierno central. De hecho, la justificación de la carrera funcionaria -el principal mecanismo weberiano para limitar la influencia del gobierno central sobre el funcionario- tiene que ver con asegurar independencia de juicio y no con fomentar la capacidad técnica. 
Por ello, el debate acerca del nivel de especialización de una agencia administrativa es conceptualmente diferente del debate acerca de su autonomía ${ }^{24}$.

Aumentar la independencia institucional es una manera de generar visibilidad, esto es, facilitar que los responsables de la agencia reciban crédito por sus aciertos y se hagan responsables de sus errores. Así, por ejemplo, el motivo para crear una Fiscalía Nacional Económica relativamente autónoma sería concentrar la responsabilidad política de combatir la formación de carteles. Mientras esa responsabilidad es asumida por el Ministro de Economía cuando se trata de organismos centralizados, bajo un esquema de relativa independencia la responsabilidad pasa a recaer en el Fiscal Nacional Económico. Teniendo en cuenta que ejercer apropiadamente la función regulatoria toma tiempo y esfuerzo, aumentar la independencia institucional puede funcionar como una manera de establecer recompensas políticas y conseguir que los reguladores hagan bien su trabajo ${ }^{25}$. De manera similar a lo que sucede con la propiedad residual en los contratos incompletos, la autonomía hace que el responsable de las decisiones responda por el resultado obtenido y no solamente por el esfuerzo desplegado para lograrlo ${ }^{26}$.

$\mathrm{Al}$ igual que con la tesis anterior, sin embargo, la visibilidad tiene también costos. Junto con fomentar la capacidad técnica del regulador, también se hace más difícil verificar el cumplimiento de sus objetivos institucionales ${ }^{27}$. A medida que la especificidad técnica de la regulación aumenta, aumentan las asimetrías de información entre la agencia regulatoria y los órganos de representación política, acentuándose con ellos los problemas de selección adversa y riesgo moral. Al igual que con la tesis de la estabilidad, la autonomía favorece que existan divergencias entre la voluntad mayoritaria y las políticas implementadas por la agencia. Por otra parte, fomentar la responsabilidad individual de una agencia lógicamente disminuye sus incentivos para colaborar con otras potenciales agencias involucradas ${ }^{28}$. Ello, a su vez, afecta la armonía del sistema

${ }^{24}$ El problema que plantea la especialización tiene que ver con que, junto con fomentar la capacidad técnica, dificulta la coordinación entre los distintos organismos que comparten competencias regulatorias. En general, los activos que cumplen una finalidad específica típicamente son difíciles de reutilizar, por lo que dicha especificidad es la que determina el tamaño de una empresa (BAwN, K., op. cit., 1995). Con las organizaciones administrativas sucede algo similar. Por ejemplo, un experto en regulación bancaria puede ser muy competente para monitorear el cumplimiento de las obligaciones de encaje de las instituciones financieras, pero seguramente le costaría mucho fiscalizar la legislación sobre protección al consumidor o del medio ambiente.

${ }^{25}$ STEPhenson, M., "Bureaucratic decisión costs and endogenous agency expertise", en Journal of Law and Economics and Organization, vol. XXIII, N ${ }^{\circ}$ 2, 2007, pp. 469-498.

${ }^{26}$ Utilizando el lenguaje de la literatura relativa a problemas de agencia, la autonomía permite introducir incentivos de alto poder en una relación formal de delegación. Para una revisión de la idea de propiedad residual en el diseño de instituciones regulatorias, con especial énfasis en procesos de privatización (ver Hart, O., Shleifer A., y Vishny, R., "The proper scope of governmente: Theory and an application to prisions", en The Quartely Journal of Economics, vol. CXII, N 4, 1997, pp. 1127-1161).

${ }^{27}$ Gailmard, S., "Expertise, subversión and bureaucratic discretion", en Journal of Law, Economics and Organization, vol. XVIII, $\mathrm{N}^{\circ} 2,2002$, pp. 563-555.

${ }^{28}$ Freeman, J. y Rossi, J., “Agency coordination in shared regulatory space”, en Harvard Law Review, vol. CXXV, N 5,2012 , pp. 1131-1211. 
regulatorio en su conjunto, especialmente en aquellos ámbitos donde se superponen las competencias de distintas agencias regulatorias ${ }^{29}$.

\section{Gradualidad, ATRINCheramiento y Desafíos De la COYUntura}

Adoptando una mirada histórica, uno puede distinguir en Chile dos grandes modelos de agencias regulatorias independientes. En primer lugar está el modelo de las autonomías constitucionales seguido por el Banco Central y la Contraloría General de la República. En segundo lugar está el modelo de las superintendencias. Ambos modelos de diseño institucional se diferencian en tres planos conceptualmente distinguibles: proceso de implementación, independencia formal y definición de competencias. Pasemos a revisar cada uno de estos ámbitos con mayor detención.

En el caso de las autonomías constitucionales, su proceso de implementación fue gradual. Según vimos al comienzo de este trabajo, tanto la Contraloría General de la República como el Banco Central formaron parte del programa de reformas impulsado por la misión Kemmerer ${ }^{30}$. Con todo, el diseño institucional de ambos organismos cambió significativamente a lo largo de los años. El Banco Central comenzó siendo precisamente eso, un banco donde concurrían como accionistas el Estado, los distintos bancos comerciales y cualquier interesado en invertir ${ }^{31}$. Luego de un conjunto de reformas intermedias, las bases de su actual diseño institucional fueron establecidas en el siguiente gran proceso de reformas con vocación internacionalista y tecnocrática, correspondiente a la denominada "revolución silenciosa" de los Chicago boys ${ }^{32}$. Tratándose de la Contraloría General de la República, su origen se encuentra en una propuesta de la misión Kemmerer para reunir los distintos organismos encargados de la fiscalización

${ }^{29}$ Esto es lo que ocurre en Chile con la regulación financiera, donde la superposición de competencias entre el Banco Central, SBIF y SERNAC hace que se produzcan dinámicas de acción colectiva. En ciertos casos ello se traduce en regulaciones contradictorias, como sucedió con las reglas referidas a modificación de comisiones para tarjetas de crédito. En otros casos, sin embargo, las distintas agencias involucradas entran en una dinámica de pasividad recíproca, como habría sucedido con la supervisión de las renegociaciones de deudas por parte de la empresa La Polar. Ver, PARdow, D., El Servicio Nacional del Consumidor de Chile (Sernac) y los regulados sectoriales: buscando mecanismos para una mejor coordinación, 2015.

${ }^{30}$ Drake, P., The money doctor in the Andes: the Kemmerer mission, 1923-1933, Duke University Press, Duke, 1989.

31 Originalmente existían tres series de acciones, buscando con ello equilibrar la capacidad de influencia del Poder Ejecutivo, la industria financiera y la generalidad del mercado. Con esta misma finalidad se establecieron posteriormente mecanismos de representación del Poder Legislativo. En el fondo, la idea detrás de este diseño institucional era permitir que los órganos directivos del banco representaran a todos los intereses en juego, fomentando así la adopción de decisiones consensuadas y estables. En términos generales, sin embargo, la literatura está conteste en el fracaso de este diseño para limitar la influencia del Poder Ejecutivo. Ver: Carrasco, C., Banco Central de Chile, 1925-1964: Una historia institucional, Banco Central de Chile, Santiago, 2009.

${ }^{32}$ Ferrada, J.C., "La autonomía como técnica de reparto de potestades públicas: El caso del Banco Central de Chile", en Revista Chilena de Derecho, Pontificia Universidad Católica de Chile, vol. XXV, 1998, pp. 335-344. 
del gasto público planteado, avanzándose posteriormente hacia una idea de autonomía constitucional durante el decenio comprendido entre 1943 y 1952. En ambos casos, sin embargo, desde un comienzo existe una búsqueda de estabilidad mediante diseños institucionales que limiten la influencia del Poder Ejecutivo. Esta idea de lograr un compromiso intertemporal que limite las oscilaciones de la política contingente está implícita en nuestro modelo de autonomías constitucionales.

Al contrario, el modelo de superintendencias pareciera haber seguido una lógica de atrincheramiento. En términos generales, la creación de superintendencias fue un complemento al programa de privatizaciones impulsado por los Chicago boys ${ }^{33}$. Junto con privatizar la empresa estatal que hasta el momento se encargaba de prestar servicios, el legislador típicamente establecía la normativa que protegía las condiciones de competencia en ese mercado y entregaba a una superintendencia específica la supervisión del mismo ${ }^{34}$. A diferencia de lo sucedido con la gradualidad en la implementación de las autonomías constitucionales, las superintendencias aparecen de manera coetánea con un cambio significativo en la orientación política de la regulación, manteniendo su diseño institucional original hasta el inicio del proceso de admisión de Chile a la OCDE.

En el ámbito de la independencia formal, el modelo de autonomías constitucionales se caracteriza por alcanzar niveles relativamente altos ${ }^{35}$. Ello que se refleja en que el Presidente de la República carece de facultades para derogar la normativa emitida por esta agencia o alterar su presupuesto anual, las autoridades superiores de la agencia son nombradas previa confirmación del Senado, y la revocación de su mandato exige la intervención de distintos Poderes del Estado ${ }^{36}$. En el caso del Banco Central, además, su dirección superior está en manos de un cuerpo colegiado. Por su parte, el modelo de superintendencias tiene una autonomía formal relativamente baja. Sus facultades normativas están subordinadas a la potestad reglamentaria del Presidente ${ }^{37}$. Además, el

33 García, J. F., op. cit., 2009, pp. 319-372.

${ }^{34}$ Ciertamente hay casos como la SVS y la SBIF, cuyo origen estuvo en recomendaciones de la misión Kemmerer. Incluso en estos casos, sin embargo, las agencias involucradas fueron objeto de reformas significativas durante la década de los 80. En todos los casos, las reformas orgánicas fueron un complemento a reformas sustantivas dirigidas a liberalizar el mercado respectivo. Algo similar ocurrió en el mercado sanitario, esta vez durante la década de los 90, donde la superintendencia del ramo surgió como complemento de la privatización impulsada durante el gobierno del presidente Frei. Ver: Oxman, S., Arredondo, J. P. y Arredondo M., Privatización del sector sanitario chileno: Análisis de un proceso inconcluso, Ediciones Cesoc, Santiago, 2000.

${ }^{35} \mathrm{La}$ independencia formal, en oposición a la independencia material o efectiva, se refiere a características del diseño institucional que resultan observables a revisar la normativa aplicable. En general, estudiar las agencias a partir de su independencia formal tiene la ventaja de facilitar su comparación, especialmente con otros países y mediante índices relativamente uniformes (v.g. Gilard, op. cit., 2002).

${ }^{36}$ Pierry, P., "La administración del Estado en la Constitución Política”, en Revista de Derecho Pontificia Universidad Católica de Valparaíso, vol. X, 1986, pp. 411-433.

37 Carmona, C., op. cit., 2005. 
gobierno tiene un control presupuestario extenso sobre la agencia, mientras que existe un grado importante de discreción para nombrar y remover a los superintendentes ${ }^{38}$.

Finalmente, en términos de la definición de competencias también existe una diferencia importante entre ambos modelos. Las atribuciones del Banco Central y la Contraloría General de la República están definidas en términos funcionales. A grandes rasgos, el primero se preocupa de proteger la integridad del sistema financiero y controlar la inflación, mientras que la segunda es la responsable de auditar el gasto público y controlar la legalidad de los actos administrativos ${ }^{39}$. Lo importante es que una agencia cuya competencia se define en términos funcionales es la principal responsable de llevar a cabo esa tarea, con independencia de la naturaleza jurídica de la entidad fiscalizada o del tipo de mercado donde se realicen las conductas. En contraste, las superintendencias siguen un sistema institucional o por "silos", donde su competencia viene definida por quién es el sujeto fiscalizado, qué tipos de industrias están involucradas, u otras consideraciones semejantes. Así, por ejemplo, en términos de la función que realizan es difícil distinguir entre una compañía de seguros y una ISAPRE. De hecho, en Chile es usual que los conglomerados financieros tengan ambos tipos de empresas. No obstante, cada una de estas entidades está sometida a la fiscalización de superintendencias diferentes. Algo similar ocurre en materia de servicios básicos: la manera en que SUBTEL establece los precios máximos de los servicios de telefonía fija es bastante similar a la manera en que determinan las tarifas de la electricidad o el agua potable, pero cada uno de estos procesos administrativos es llevado adelante por una agencia diferente.

Esta distinción en materia de competencias es doblemente importante. Por una parte, atribuir competencias en términos funcionales es una manera de evitar su captura por intereses particulares ${ }^{40}$. Cuando se supervisan varios mercados y tipos de entidades simultáneamente, los intereses afectados por la regulación tienden a ser más variados y heterogéneos. Al contrario, cuando la agencia se focaliza en un solo mercado regulado como ocurre en el modelo de superintendencias, existen básicamente dos intereses en juego: el interés homogéneo de las empresas incumbentes y el interés difuso de los consumidores. Además de aumentar el riesgo de captura, los costos de la autonomía también tienden a ser mayores en un sistema de silos. Definir la competencia en atención a la naturaleza jurídica de la entidad o el tipo de industria supone el riesgo de que entidades o industrias similares enfrenten reglas diferentes ${ }^{41}$. En este contexto, resulta clave la función

${ }^{38}$ Ciertamente esta discreción ha disminuido progresivamente desde el establecimiento del sistema de Alta Dirección Pública, pero incluso en su actual versión el modelo de superintendencias tiene un nivel de independencia significativamente menor que el de las agencias regulatorias de otros países de la OCDE (GARCía, J.F. y Cordero, L., "De las superintendencias a las agencias regulatorias independientes en Chile: Aspectos constitucionales y de diseño regulatorio”, en Anuario de Derecho Público, Universidad Diego Portales, vol. II, 2010, pp. 415-435).

${ }^{39}$ Cordero, L., Lecciones de Derecho Administrativo, Thomson Reuters, Santiago, 2015.

${ }^{40}$ MACEY, J., "Organizational design and political control of administrative agencies", en Journal of Law, Economics and Organization, vol. XVIII, 1992, pp. 93-110.

${ }^{41}$ Siguiendo con los ejemplos anteriores, las reglas acerca del consentimiento tácito en los contratos de seguros son sustancialmente diferentes a las que se aplican respecto de los contratos de planes ISAPRE 
del gabinete presidencial para coordinar las distintas agencias regulatorias. Como lo señala el último reporte de la OCDE respecto de nuestra institucionalidad regulatoria, limitar la influencia presidencial estableciendo mecanismos de independencia formal supone también hacer más difícil esta labor de coordinación.

Tomando en cuenta los tres planos de comparación revisados anteriormente, la coyuntura muestra desafíos importantes. El actual proceso de reformas institucionales comenzó a mediados de la década pasada, y un primer aspecto llamativo es que nuestros diseños institucionales han dejado de seguir un patrón claramente establecido. Tratándose del Consejo para la Transparencia o del TDLC, por ejemplo, pareciera haberse replicado el diseño del Banco Central, pero estableciendo su autonomía a un nivel simplemente legal y reduciendo ciertos estándares de independencia formal ${ }^{42}$. En contraste, las reformas a la FNE o el SERNAC están mucho más cercanas a la orientación unipersonal y supeditada a control presidencial del modelo de superintendencias, claro que con una definición funcional de competencias. Más allá de relativizar cuáles son los elementos sistemáticos de nuestros modelos de diseño institucional, el principal problema de este camino híbrido es que puede terminar incurriendo en muchos de los costos de la autonomía, sin conseguir sus beneficios. Veamos por qué.

En relación con los mecanismos para limitar la influencia del Poder Ejecutivo en las decisiones de la agencia, ciertos espacios del debate público han instalado una idea minimalista para este proceso de reformas: el principal problema del modelo de superintendencias estaría en sus estándares de independencia formal. Por ello, bastaría con agregarles organismos de dirección colectivos, así como fortalecer los mecanismos de nombramiento y control presupuestario, replicando en parte el modelo de las autonomías constitucionales ${ }^{43}$. Este minimalismo, sin embargo, olvida un par de cosas. Para empezar, la importancia de separar conceptualmente estabilidad y atrincheramiento. Como muestra la literatura, aumentar la autonomía institucional en un mercado cuya legislación de fondo está políticamente sesgada únicamente disminuye el bienestar social, ya que la autonomía genera mayores costos de transacción y evita que la regulación económica refleje la posición del votante mediano. En nuestro país, el modelo de superintendencias fue implementado bajo una realidad carente de toda legitimidad democrática y comprometida con una ideología determinada, por lo que resulta difícil asumir que la legislación de fondo en materia de seguros de salud, administración de pensiones o servicios básicos refleja un consenso real en la sociedad. De hecho, una

\footnotetext{
(PARDow, op. cit., 2015). Del mismo modo, pese a aplicar el mismo modelo conceptual de empresa eficiente, los criterios sobre plusvalía o indivisibilidad varían dependiendo de la industria que se trate (FUENTES, F. y SaAvedra, E., "Problemas de implementación de la empresa eficiente: Plusvalía, indivisibilidades y obsolescencia”, en Documentos de Trabajo, Universidad Alberto Hurtado, disponible en: http://fen.uahurtado. cl/wp-content/uploads/2010/07/inv192.pdf, 2007.

42 Cordero, E., "La administración del estado en Chile y el concepto de autonomía”, en MendozA, R. (ed.), La Contraloría General de la República. 85 años de vida institucional (1927-2012), Santiago, 2012.

43 V.g., García, J. F. y Verdugo, S., "De las superintendencias a las agencias regulatorias independientes en Chile: Aspectos constitucionales y de diseño regulatorio”, en Actualidad Jurídica, Universidad del Desarrollo, vol. XXII, 2010, pp. 236-305.
} 
revisión del trabajo que distintos grupos de expertos han realizado en los últimos años muestra que lo que necesitamos son agendas largas, que cuestionen nuestro modelo de desarrollo y la manera en que queremos organizar los mercados ${ }^{44}$.

Enseguida, nuestro actual esquema de distribución de competencias responde a la idiosincrasia del programa de privatizaciones, donde las distintas industrias involucradas fueron siendo objeto de liberalización de manera progresiva. Al abrirse un proceso de reformas resulta lógico preguntarse si este esquema, que pareciera haberse ido construyendo sobre la marcha, tiene algún sentido sistemático. La evidencia disponible, en cualquier caso, tampoco es concluyente. Existe cierta tendencia en otros países a crear reguladores integrados, pero son iniciativas recientes y los motivos detrás de las mismas parecieran ser igualmente contingentes ${ }^{45}$. En este sentido, resulta interesante recoger nuestra experiencia en la creación de la Comisión de Mercados Financieros. Siguiendo las recomendaciones de la Comisión Desormeaux, la Ley No 21.000 reemplazó a la SVS con una agencia regulatoria cuya dirección queda en manos de un cuerpo colegiado, donde sus miembros son nombrados de manera similar a los consejeros del Banco Central. A lo anterior se suma el actual proyecto de ley para reformar la Ley General de Bancos, que incorpora a la SBIF dentro de la referida comisión y consolida el perímetro regulatorio de ambas superintendencias. En definitiva, se avanza simultáneamente en fortalecer la independencia formal de nuestras agencias y racionalizar el sistema de distribución de competencias.

\section{Conclusiones}

La incorporación de Chile a la OCDE marca el inicio de un tercer proceso de reformas institucionales en menos de un siglo. Al igual que las reformas impulsadas por la misión Kemmerer durante los años 20 y la "revolución silenciosa" en la década de los 80, nuestro actual proceso de reformas tiene una vocación internacionalista y tecnocrática. No obstante, es importante también marcar las diferencias a la hora de sacar lecciones atinentes a nuestra experiencia institucional. Las reformas kemmererianas fueron graduales, moviéndose progresivamente hacia un diseño con altos estándares de independencia formal y un sistema funcional de distribución de competencia. Al contrario, las reformas de los Chicago boys fueron un complemento del programa de privatizaciones, estableciendo una autoridad regulatoria diferenciada para cada uno de los mercados que pasaron a liberalizarse. Ello generó un sistema atomizado y con bajos niveles de independencia formal, donde existe una multiplicidad de reguladores relativamente dependiente del gobierno para cada industria específica.

${ }^{44}$ SAavedra, E., op. cit., 2014.

45 En el caso español, por ejemplo, la creación de un regulador integrado para los distintos servicios básicos habría estado motivada en la necesidad de disminuir el tamaño del Estado y reducir gasto fiscal, antes que en razones sistemáticas (v.g. Ortiz, S., "Apuntes sobre la Comisión Nacional de los Mercados y la Competencia de España”, en Revista Digital de Derecho Administrativo, vol. XIV, 2015, pp. 237-254). 
Ciertamente la independencia favorece la estabilidad de las reglas del juego y fomenta la especialización de nuestros reguladores. Ahora bien, es importante recordar que también tiene costos. La estabilidad es una solución razonable cuando las reglas subyacentes reflejan un consenso real en la sociedad. En caso contrario, la independencia institucional erosiona la legitimidad democrática de nuestras reglas, transformándose en una barrera para los cambios que quieren las mayorías políticas. La especialidad, por su parte, tiende a fragmentar la regulación económica. Cuanto más especialistas se vuelven las agencias, son también más difíciles de fiscalizar, más sencillas de capturar por los regulados y menos dispuestas a colaborar entre sí. Un diseño institucional apropiado debe ponderar los costos y beneficios de la independencia, evaluando críticamente nuestra experiencia previa.

En este contexto, enfocarse en aumentar los niveles de independencia formal de las actuales superintendencias parece derechamente una mala idea. Nuestro actual sistema de regulación por silos genera espacios para el arbitraje regulatorio, fomenta dinámicas de acción colectiva y descuida los aspectos conductuales de la regulación. Ciertamente existen beneficios en generar agencias especializadas a nivel de industria o entidad, pero ellos deben contraponerse con el mayor riesgo de captura que lleva asociado, así como con los costos de coordinación de un sistema fragmentado. En definitiva, es importante que el aumento en los niveles de independencia venga acompañado de una racionalización de las competencias regulatorias y un fortalecimiento de los mecanismos de coordinación administrativa. Desde esta perspectiva, la reforma que crea la actual Comisión de Mercados Financieros apunta en la dirección correcta, reuniendo bajo una sola autoridad la regulación de los bancos comerciales, las compañías de seguros y el mercado de valores.

\section{BIBLIOGRAFÍA}

BAwn, K., "Political control versus expertise: Congressional choices about administrative procedures”, en American Political Science Review, vol. LXXXIX, 1995, pp. 62-73.

Carmona, C., Una aproximación general sobre las superintendencias desde la perspectiva del Derecho, [en consulta con el autor], Santiago, 2005.

Carrasco, C., Banco Central de Chile, 1925-1964: Una historia institucional, Banco Central de Chile, Santiago, 2009.

Cordero, E., "La administración del Estado en Chile y el concepto de autonomía”, en Mendoza, R. (ed.), La Contraloría General de la República. 85 años de vida institucional (1927-2012), Santiago, 2012.

Cordero, L., Lecciones de Derecho Administrativo, Thomson Reuters, Santiago, 2015.

Drake, P., The money doctor in the andes: the Kemmerer misión, 1923-1933, Duke University Press, Duke, 1989.

Ferrada, J.C., "La autonomía como técnica de reparto de potestades públicas: El caso del Banco Central de Chile", en Revista Chilena de Derecho, Pontificia Universidad Católica de Chile, vol. XXV, 1998, pp. 335-344.

Freeman, J. y Rossi, J., "Agency coordination in shared regulatory space", en Harvard Law Review, vol. CXXV, No 5, 2012, pp. 1131-1211. 
Fuentes, F. y SaAvedra, E., Problemas de implementación de la empresa eficiente: Plusvalía, indivisibilidades y obsolescencia, en Documentos de Trabajo, Universidad Alberto Hurtado, disponible en: http://fen.uahurtado.cl/wp content/uploads/2010/07/inv192.pdf, 2007.

GAILMARD, S., "Expertise, subversión and bureaucratic discretion", en Journal of Law, Economics and Organization, vol. XVIII, $\mathrm{N}^{\circ} 2,2002$, p. 563-555.

García, J. F. y Verdugo, S., "De las superintendencias a las agencias regulatorias independientes en Chile: Aspectos constitucionales y de diseño regulatorio", en Actualidad Jurídica, Universidad del Desarrollo, vol. XXII, 2010, pp. 236-305.

García, J. F., “¿Inflación de superintendencias? Un diagnóstico crítico desde el derecho regulatorio”, en Actualidad Jurídica, Universidad del Desarrollo, vol. XIX, 2009, pp. 327-330.

García, J. F. y Cordero, L., "De las superintendencias a las agencias regulatorias independientes en Chile: Aspectos constitucionales y de diseño regulatorio", en Anuario de Derecho Público, Universidad Diego Portales, vol. II, 2010, pp. 415-435.

Gersen, J., "Designing agencies”, en Farber, D. and O'Connell, A. J., Cheltenham Research handbook on public choice and public law, Edward Elgar Publishing, Reino Unido, 2010, pp. 333-362.

GiLARDI, F., "Policy credibility and delegation to independent regulatory agencies: A comparative empirical analysis", en Journal of European Public Policy, vol. IX, No 6, 2002, pp. 873-893.

Hart, O., Shleifer, A., y Vishny, R., "The proper scope of governmente: Theory and an application to prisions", en The Quartely Journal of Economics, vol. CXII, No 4, 1997, pp. 1127-1161.

Hunneus, C., "Technocrats and politicians in an authoritarian regime: The 'ODEPLAN boys' and the 'gremialists' in Pinochet's Chile", en Journal of Latin American Studies, vol. 32, $\mathrm{N}^{\circ} 2,2000$, pp. 450-461.

Issacharoff, S. y Miller, L., "Democracy and electoral processes", en Farber, D. y O'Connel A.J. (eds.), Research handbook on public choice and public law, Edward Elgar, Cheltenham, 2010, pp. 221-258.

Jellinek, G., Teoría general del Estado, Fernando de los Ríos [trad.], Fondo de Cultura Económica, México, 2012.

LEVY, B., Regulations, institutions and commitment: Comparative studies of telecomunications, Cambridge University Press, Cambridge, 2008.

LEWIS, D., Presidents and the politics of agency design: Political insulation in the United States government bureaucracy, 1946-1997, Stanford University Press, Standford, 2004.

LüDERs, R., La misión Klein-Saks, los Chicago boys y la política económica, en Documentos de trabajo, $N^{\circ}$ 411, Instituto de Economía, Pontificia Universidad Católica de Chile, 2012, pp. $1-34$.

MACEY, J., "Organizational design and political control of administrative agencies", en Journal of Law, Economics and Organization, vol. XVIII, 1992, pp. 93-110.

Majone, G., "Nonmajoritarian institutions and the limits of democratic governance: a political transaction-cost approach", en Journal of Institutional and Theoretical Economics, vol. CLVII, 2001, pp. 57-78.

McCubbins, M., "The legislative design of regulatory structure”, en American Journal of Political Science, vol. XXXIX, No 4, 1985, pp. 721-748.

Morandé, F. y Díaz, J. D., "Chile en el club de los países desarrollados: beneficios, oportunidades y desafíos del ingreso a la OCDE”, en Morandé, F. y Díaz, J. D. (eds.), Chile en el club de los países desarrollados, Ediciones Universidad Católica, Santiago, 2010.

NoRTH, D., Institutions, institutional change and economic perfomrance, Cambridge University Press, Cambridge, 1990.

Ogus, A., Regulation, legal reform and economic theory, Clarendon Press, Oxford, 1994, pp. 181 y ss. 
Ortiz, S., "Apuntes sobre la Comisión Nacional de los Mercados y la Competencia de España”, en Revista Digital de Derecho Administrativo, vol. XIV, 2015, pp. 237-254.

Oxman, S., Arredondo, J. P. y Arredondo M., Privatización del sector sanitario chileno: Análisis de un proceso inconcluso, Ediciones Cesoc, Santiago, 2000.

PARDow, D., El Servicio Nacional del Consumidor de Chile (Sernac) y los regulados sectoriales: buscando mecanismos para una mejor coordinación, 2015.

Pierry, P., "La administración del Estado en la Constitución Política", en Revista de Derecho Pontificia Universidad Católica de Valparaíso, vol. X, 1986, pp. 411-433.

Ripert, G., Aspectos jurídicos del capitalismo moderno, Comares, Granada, 2001, pp. 281.

SAAVEdRa, E., "El modelo económico-político de Chile: Desarrollo institucional en la encrucijada", en Economía y Política, Universidad Adolfo Ibáñez, vol. I, 2014, pp. 117-178.

Silva, P., In the name of reason: Technocrats and politics in Chile, PSU Press, Pennsylvania, 2010.

STEPhenson, M., "Bureaucratic decisión costs and endogenous agency expertise", en Journal of Law and Economics and Organization, vol. XXIII, N ${ }^{\circ} 2,2007$, pp. 469-498.

STIGLER, G., "The theory of economic regulation", en Bell Journal of Economics and Management Science, vol. II, 1971, pp. 3-21, 1971.

Tirole, J., "The internal organization of government", en Oxford Economic Papers, 1994, pp. 1-29.

Weber, M., Economía y Sociedad, Fondo de Cultura Económica, México, 1956, pp. 382-394.

Wilson, J., Bureaucracy: What government agencies do and why they do it, Basic Book, Nueva York, 1989, pp. 72-82.

Wood, D. у Boothe, J., "Political transaction costs and the politics of administrative design", en Journal of Politics, vol. LXIV, 2004, pp. 176-202. 
